\title{
Model-Based Engine Fault Detection Using Cylinder Pressure Estimates from Nonlinear Observers
}

\author{
Minghui Kao and John J. Moskwa \\ Powertrain Control Research Laboratory \\ Department of Mechanical Engineering \\ University of Wisconsin-Madison \\ 1513 University Avenue \\ Madison, WI 53706, USA. \\ Email: moskwa@engr.wisc.edu
}

\begin{abstract}
There are two indicated torque input observers presented in paper. The input estimation problem is transformed into the control tracking problem. The sliding mode control with integrator and PI with feedforward control are used for tracking control blocks. The indicated torque estimations are applied to detect engine firing faults. Cylinder pressure centroid and cylinder pressure difference approaches for engine diagnostics are also discussed in this paper. Simulation results for a six-cylinder engine with abnormal combustion are plotted to show engine combustion problems.
\end{abstract}

\section{Introduction}

The purpose of this paper is to investigate the use of cylinder pressure and combustion torque estimations for engine diagnostics. Meeting the on-board diagnostics requirements have been a challenge for automotive industry. Misfire detection is a principal goal in this challenge. There have been researchers pursuing system diagnostics in past decades for different engineering disciplines $[2,3,11]$, such as diagnostics for chemical or nuclear reactors. However, many diagnostic techniques are still emerging and being developed. The nonlinear model-based approach is especially suitable for engine diagnostics because most of the engine processes are inherently nonlinear. Addressing this issue using nonlinear model-based observers can be very intuitive by estimating important engine physical variables directly (cylinder pressure indicated torque, theoretical fuel burning rate, efficiency, etc.).

Since the power of an engine which comes from combusting the air-fuel mixture drives the piston crankshaft assembly, valuable information (engine speed, acceleration, cylinder head bolt stretch, vibration signature, etc.) that is related to or affected by the combustion process can be used to estimate combustion quality $[1,10]$. A nonlinear varying inertia crankshaft model is used to account for the engine speed fluctuations that are caused by inertial torques, and to provide the input indicated torque observers an adequate nominal model of the process. Although a constant inertia crankshaft model is sufficient for typical engine dynamic mean performance simulation, it can hardly be used to relate engine indicated torque and combustion faults because of its low bandwidth characteristics. On the other hand, the finite element compliance crankshaft model is too complicated to be used in this dynamic observer approach for on-board diagnostics and there are effective means of estimating cylinder pressure without this addition. A combustion pressure dynamic equation is used in the cylinder pressure observer [6] to simulate the pressure pulses and provide the cylinder pressure observer a nonlinear dynamic correcting feedforward term that makes the pressure estimates converge faster and more accurately. The indicated torque estimation using a sliding cylinder pressure observer, given in $[5,6]$, is included here for comparison.

There are two input indicated torque observers presented in this paper. Designs of a sliding input indicated torque observer and a PI input indicated torque observer will be outlined and discussed. The input indicated torque estimation problem has been investigated by Srinivasan et al. [14] and Zhang and Rizzoni [15]. However, the nonlinear inertia effect is not explicitly explored, and this leads to very large estimation errors at high engine speed. The sliding observer and control methods are selected in this study because of their robustness. convergence, and accuracy. The estimation problem is first transformed into the control tracking problem. Controllers are used to force the estimated speed to track the measured varying speed. The sliding controller varies the estimated indicated torque in order to decrease the speed estimation error (estimated speed minus measured speed), and convergence is guaranteed by the Lyapunov-like function. The nonlinear engine crankshaft dynamic model defines the relationships between speed error and the indicated torque estimate. These sliding and PI input indicated torque observers have the advantage that they can be used at high engine speeds, since the inertial forces are accurately estimated at all engine speeds, and can predict both transient and steady state behaviors. Large inertial force errors, caused by the highly nonlinear nature of engine geometry, limit the usefulness of other cylinder pressure estimation schemes.

This paper also discusses the use of the estimated cylinder pressure profile, obtained from the observer, to identify engine faults (include misfire and other conditions). The cylinder pressure dynamic equation is solved analytically to study the relationship between fuel burning rate and cylinder pressure. The locality and magnitude of a cylinder pressure centroid can be related to fuel injection timing and duration [9]. The cylinder pressure difference approaches (firing pressure minus motoring pressure, and pressure difference between cylinder pressures before and after TDC) are being studied and related

This research is supported by funding from Cummins Engine Company, Inc.. 
to the combustion heat release. Also, the engine peak cylinder pressure can be used for engine control and related to engine combustion power and fault detection. Application of these cylinder pressure profile approaches and observers to engine diagnostics are inciuded. Finally simulation results will be used to support these designs.

\section{Input Indicated Torque Observers}

Cylinder pressure force that is applied to the moment arm of piston connecting-rod assembly results to the indicated torque. The varying indicated torque information can be useful in engine cylinder-by-cylinder fuel management and diagnostics. Indicated torque is a control input to drive the engine's varying speed dynamics. Fuel injection and timing are controlled to track desired engine speed and load profiles. The estimation can be transformed into a control tracking problem (i.e., for estimated speed to track the measured speed). A good tracking controller block design then guarantees a good input estimation.

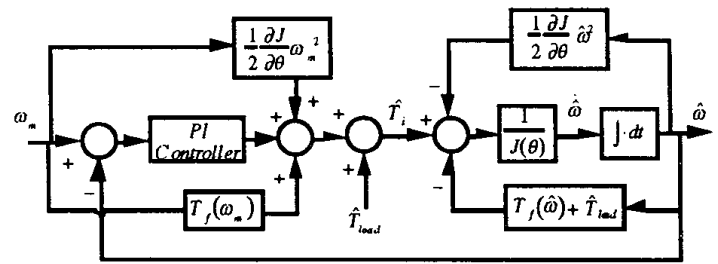

Figure 1: Block Diagram of a PI Feedforward Input Indicated Torque Observer.

PI Feedforward Input Observer

Figure 1 shows the block diagram of a PI input observer. The block from indicated torque $\hat{T}_{i}$ to estimated engine speed $\hat{\omega}$ outlines the nonlinear engine crankshaft dynamics. Nonlinear feedforward terms $\hat{T}_{f}\left(\omega_{m}\right)$ and $(1 / 2)(\partial J / \partial \theta) \omega_{m}{ }^{2}$ are used to ideally decouple the nonlinearity of crankshaft dynamic block. A PI controller is used to force the engine varying speed estimate to follow the measured engine speed. Open loop dynamics of the crankshaft are given in (1) assuming nonlinearity is perfectly decoupled (which is not always true, and the PI controller is used to correct speed tracking error). Therefore, the closed loop characteristic equation can be derived as in (2).

$$
\begin{aligned}
& \frac{\hat{\omega}}{\hat{T}_{i}}=\frac{1}{s \cdot J(\theta)} \\
& 1+\left(k_{p}+\frac{k_{i}}{s}\right) \frac{1}{s \cdot J(\theta)}=0 \\
& s^{2}+\frac{k_{p}}{J(\theta)} s+\frac{k_{i}}{J(\theta)}=0
\end{aligned}
$$

The gains of the PI controller as shown in (3) can be derived by placing the closed loop poles at $\lambda_{1}$ and $\lambda_{2}$.

$$
\begin{aligned}
& k_{r}=-J(\theta)\left(\lambda_{1}+\lambda_{2}\right) \\
& k_{i}=J(\theta) \cdot \lambda_{1} \cdot \lambda_{2}
\end{aligned}
$$

The input indicated torque can then be obtained by adding the nonlinear feedforward terms and PI control output as in (4).

$$
\begin{aligned}
\hat{T}_{i}= & \hat{T}_{f}\left(\omega_{m}\right)+\hat{T}_{\text {load }}+\frac{1}{2} \frac{\partial J}{\partial \theta} \omega_{m}{ }^{2} \\
& +k_{p}\left(\omega_{m}-\hat{\omega}\right)+k_{i} \int\left(\omega_{m}-\hat{\omega}\right) d t
\end{aligned}
$$

The nonlinear feedforward decoupling greatly improves the tracking and estimation abilities. A good indicated torque estimation is achieved.

\section{Sliding Input Observer with Integrator}

Figure 2 shows a block diagram of the sliding input indicated torque observer with integrator. The sliding controller has very good tracking performance and the integrator is included to eliminate the steady state error in tracking. Nonlinear terms $\hat{T}_{f}(\hat{\omega})$ and $(1 / 2)(\partial J / \partial \theta) \hat{\omega}^{2}$ are fed to the summation point before $\hat{T}_{i}$ by using the estimated speed information. The sliding surface is chosen as the combination of the speed estimation difference and it integral.

$S=\left(\frac{d}{d t}+\lambda\right) \int_{0}^{t}\left(\hat{\omega}-\omega_{m}\right) d t=\left(\hat{\omega}-\omega_{m}\right)+\lambda \int_{0}^{t}\left(\hat{\omega}-\omega_{m}\right) d t$

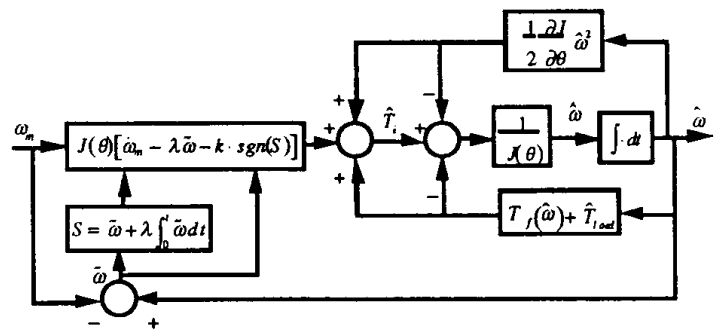

Figure 2: Block Diagram of a Sliding Input Indicated Torque Observer.

In order to satisfy the sliding condition (6), the derivative of sliding surface $(\dot{S})$ is selected as in (7)

$\operatorname{sgn}(S) \dot{S} \leq-\eta$

$\dot{S}=\left(\dot{\hat{\omega}}-\dot{\omega}_{m}\right)+\lambda\left(\hat{\omega}-\omega_{m}\right)=-k \cdot \operatorname{sgn}(S)$

$\dot{\hat{\omega}}=\left[\hat{T}_{i}-T_{f}(\hat{\omega})-\hat{T}_{\text {load }}-\frac{1}{2} \frac{\partial J}{\partial \theta} \hat{\omega}^{2}\right] / J(\theta)$

Crankshaft dynamics (8) are substituted into (7), and the indicated torque estimation (9) can be derived.

$$
\begin{aligned}
\hat{T}_{\mathrm{i}}= & T_{f}(\hat{\omega})+\hat{T}_{\text {load }}+\frac{1}{2} \frac{\partial J}{\partial \theta} \hat{\omega}^{2} \\
& +J(\theta) \cdot\left[\dot{\omega}_{m}-\lambda\left(\hat{\omega}-\omega_{m}\right)-k \cdot \operatorname{sgn}(S)\right]
\end{aligned}
$$

The closed loop speed tracking dynamics (10) (from measured speed to the estimated speed) is given by substituting (9) into (8).

$$
\begin{aligned}
\dot{\hat{\omega}} & =\left[\hat{T}_{i}-T_{f}(\hat{\omega})-\hat{T}_{\text {load }}-\frac{1}{2} \frac{\partial J}{\partial \theta} \hat{\omega}^{2}\right] / J(\theta) \\
& =\dot{\omega}_{m}-\lambda\left(\hat{\omega}-\omega_{m}\right)-k \cdot \operatorname{sgn}(S)
\end{aligned}
$$

The switching gain $k$ should be selected greater than $\eta$ and much greater than the modeling error to guarantee speed of convergence and robustness. The gain $k$ determines how fast the system converges to the sliding mode. The value of $\lambda$, which like the eigenvalue of linear systems, defines the dynamics inside the reduced order dynamics of the sliding mode. Notice that this sliding input observer requires the acceleration information which can be calculated by using 
numerical differentiation. Although numerical differentiation will introduce errors into the indicated torque estimation, the sliding control is robust enough and will take care of part of that error.

\section{Pressure Estimates for Engine Fault Detection}

Cylinder pressure that is generated by engine combustion contains much information related to engine performance such as fuel burning rate, combustion heat release rate, and fuel-air ratio. Fuel-air ratio estimation using cylinder pressure has been discussed in $[4,13]$. Powell et al. [12] investigated an analysis model for cylinder pressure feedback control. In this section, several cylinder pressure data manipulations are discussed for the purpose of engine fault detection (especially misfire detection).

Cylinder peak pressure can be used to indicate how well the engine performed, and is an important factor for engine design. Powell [13] discussed the use of peak cylinder pressure for spark ignition engine control. The locality and magnitude of cylinder pressure profile centroid can be used to indicate misfire [9]. The non-firing cylinder pressure profile is subtracted from the firing cylinder pressure profile. The centroid of pressure profile difference approach amplifies the combustion symptom, and can be related to the injection crankangle and injected fuel mass. However, this approach needs to be accompanied with an empirically determined database.

\section{Cylinder Pressure Centroid}

The cylinder pressure observer discussed in $[6,8]$ is used to estimate cylinder pressure. The location of pressure centroid in terms of crankangle is given as

$C \theta=\frac{\int P \cdot \theta \cdot d \theta}{\int P \cdot d \theta}$

Table 1 gives descriptions for simulation cases that are used to compare the centroid locations of simulated actual pressure and observed cylinder pressure profiles. Simulated actual data is obtained by running the detailed one-cylinder, cylinder-bycylinder model, and simulated observed data is from cylinder pressure observer that is designed by using simplified cylinderby-cylinder model [7]. The sliding cylinder pressure observer has the form [6]:

$$
\begin{aligned}
\dot{\hat{\omega}}=[ & \sum_{i=1}^{n} 1000 \hat{P}_{c y l i} \frac{d V_{i}}{d \theta}-\hat{T}_{f}-\hat{T}_{\text {lowd }} \\
- & \left.\frac{1}{2} \frac{\partial J}{\partial \theta} \hat{\omega}^{2}\right] / J(\theta)-\alpha_{1} \tilde{\omega}-k_{1} \operatorname{sgn}(\tilde{\omega}) \\
\dot{\hat{P}}_{c y l i} & =\frac{\gamma-1}{V}\left(\hat{\dot{m}}_{\text {shum }} Q_{L H V}+\dot{\hat{Q}}_{h t}\right)-\frac{\hat{P}_{c l^{l} i} \gamma}{V} \dot{V} \\
& -\alpha_{c y l i} \tilde{\omega}-k_{c y l i} \operatorname{sgn}(\tilde{\omega})
\end{aligned}
$$

The simulation results for centroid comparisons are documented in Table 2. The observed centroids $C \theta_{\text {ob }}$ are very close to the simulated actual pressure centroids $C \theta_{\text {art }}$ for different conditions. Table 2 (a) and (b) give the centroids that have the specified range (including TDC). The misfired combustion has a cylinder pressure centroid close to 0 degree because misfired cylinder pressure is almost symmetry about TDC. Tables 1 and 2 show that the pressure centroid method can be used for misfire detection.
Table 1: Different Engine Running Conditions

\begin{tabular}{|c|c|}
\hline case \#1 & $1606 \mathrm{RPM}$, normal combustion \\
\hline case \#2 & $1223 \mathrm{RPM}$, normal combustion \\
\hline case \#3 & $1001 \mathrm{RPM}$ normal combustion \\
\hline case \#4 & 1567 RPM misfire \\
\hline
\end{tabular}

Table 2: Simulated Actual and Observed Cylinder Pressure Centroids.

(a) $-60 \leq \theta \leq 60$

\begin{tabular}{|c|c|c|}
\hline \multicolumn{3}{|c|}{ (a) $-60 \leq \theta \leq 60$} \\
\hline Case \# & $C \theta_{\text {act }}$ & $C \theta_{o b}$ \\
\hline 1 & 6.20 & 5.91 \\
\hline 2 & 6.59 & 6.65 \\
\hline 3 & 6.64 & 6.27 \\
\hline 4 & -0.15 & 0.54 \\
\hline
\end{tabular}

(b) $-120 \leq \theta \leq 120$

\begin{tabular}{|c|c|c|}
\hline Case \# & $C \theta_{a c t}$ & $C \theta_{o b}$ \\
\hline 1 & 8.96 & 8.87 \\
\hline 2 & 9.5 & 9.98 \\
\hline 3 & 9.58 & 9.37 \\
\hline 4 & -0.24 & 0.76 \\
\hline
\end{tabular}

Cylinder Pressure Difference

The cylinder pressure dynamic equation is given in (14). It is a first order nonlinear differential equation, and can be solved analytically [5].

$$
\dot{P}_{c y l i}=\frac{\gamma-1}{V}\left(\dot{m}_{f h u r n} Q_{L H V}+\dot{Q}_{h t}\right)-\frac{P_{c y l i} \gamma}{V} \dot{V}=f_{c y l i}
$$

The solution of a nonlinear first order differential equation (15) is given in (16) and (17).

$$
\begin{aligned}
& y^{\prime}+f(x) y=g(x) \\
& y=e^{-h(x)}\left[\int e^{h(x)} g(x) d x+c\right] \\
& h(x)=\int f(x) d x
\end{aligned}
$$

The value of $c$ can be determined by using initial conditions. A simplified solution of $\int e^{h(x)} g(x) d x$ can be obtained by assuming constant combustion volume $V_{\text {cons }}$

$$
\begin{gathered}
\int e^{h(x)} g(x) d x=\int V^{\gamma} \frac{\gamma-1}{V}\left(\dot{m}_{f u w n} Q_{L H V}+\dot{Q}_{H s}\right) d t \\
=(\gamma-1) \int V^{\gamma-1}\left(\dot{m}_{f a w n} Q_{L H V}+\dot{Q}_{h t}\right) d t \\
=(\gamma-1) V_{c o m b}^{\gamma-1}\left(m_{f b u r n} Q_{L H V}+Q_{h t}\right)
\end{gathered}
$$

The cylinder pressure is solved and shown in (19). The term $V^{-\gamma} P_{B D C} V_{B D C}^{\gamma}$ in this cylinder pressure equation (19) represents a polytropic compression or expansion. The first term in (19) accounts for the fuel combustion and heat transfer loss.

$$
\begin{aligned}
& P_{c y l i}=V^{-\gamma}\left[(\gamma-1) V_{c c o m b}^{\gamma-1}\left(m_{\text {fbw } n} Q_{L H V}+Q_{h s}\right)+c\right] \\
& =V^{-\gamma}(\gamma-1) V_{c \text { comb }}^{\gamma-1}\left(m_{f b w n} Q_{L H V}+Q_{h r}\right)+V^{-\gamma} P_{B D C} V_{B D C}^{\gamma}
\end{aligned}
$$

The firing cylinder pressure content is mostly related to the combustion and fuel heat release. Therefore, the pressure difference is a good source for engine combustion information and misfire detection. The $\Delta \boldsymbol{P}_{\text {fire }}$ is the pressure difference between fired and misfired cylinder pressures, and is given in (20). 


$$
\begin{aligned}
\Delta P_{\text {fire }} & =P_{\text {fire }}-P_{\text {misfire }} \\
& =V^{-\gamma}(\gamma-1) V_{\text {comb }}^{\gamma-1}\left(m_{\text {flurn }} Q_{L H V}+Q_{h t}\right)
\end{aligned}
$$

The non-firing cylinder pressure data for comparing the firing cylinder pressure should be either generated by a simulation program or experiment data.

The $\Delta P_{d e g}$ is the cylinder pressure difference between pressures after and hefore TDC at selected crankangle, and is shown in (21).

$$
\begin{aligned}
\Delta P_{d e g} & =P_{d c g}-P_{-d e g} \\
& =V_{d e g}^{-\gamma}(\gamma-1) V_{c o m b l}^{\gamma-1}\left(m_{f l u r n} Q_{L H V}+Q_{h t}\right)
\end{aligned}
$$

The cylinder pressure differences in (20) and (21) are strongly affected by the amount of fuel burned. The partial derivatives of (20) and (21) are given in (22). The engine combustion health monitoring approach can use $\Delta P$ values from cylinder to cylinder and cycle to cycle, and compare them with the fuel mass curves of every cylinder.

$$
\begin{aligned}
& \frac{\partial \Delta P_{\text {fire }}}{\partial m_{\text {flurn }}}=V^{-\gamma}(\gamma-1) V_{\text {comb }}^{\gamma-1} Q_{L H V} \\
& \frac{\partial \Delta P_{\text {ckg }}}{\partial m_{\text {flurn }}}=V_{d k g}^{-\gamma}(\gamma-1) V_{c o m b}^{\gamma-1} Q_{L H V}
\end{aligned}
$$

\section{Simulation Results}

Table 3: Descriptions of Various Observers for Indicated Torque Estimations.

\begin{tabular}{|c|c|}
\hline PIIO_I & $\begin{array}{c}\text { PI Input Observer Using Constant } \\
\text { Crankshaft Inertia }\end{array}$ \\
\hline PIIO_J & $\begin{array}{c}\text { PI Input Observer Using Varying } \\
\text { Crankshaft Inertia }\end{array}$ \\
\hline SIO & Sliding Input Observer with Integrator \\
\hline CPO & Sliding Cylinder Pressure Observer \\
\hline
\end{tabular}

Table 4: Estimation Errors of Various Input Indicated Torque

\begin{tabular}{|c|c|}
\hline \multicolumn{2}{|c|}{ Observers. } \\
\hline Estimators & Mean Error (N.m) \\
\hline PIIO_I & 61.8 \\
\hline PIIO_J & 47.9 \\
\hline SIO & 3.7 \\
\hline CPO & 14.1 \\
\hline PIIO_I Noise & 122.5 \\
\hline PIIO_J Noise & 125.0 \\
\hline SIO Noise & 113.2 \\
\hline CPO Noise & 27.1 \\
\hline
\end{tabular}

There are four different observer schemes that are used in the simulation to estimate the indicated torque. The noise of 400 $\mathrm{Hz}$ is chosen to represent first mode of crankshaft oscillation in the Cummins L10 engine due to the compliant crankshaft. The cases with speed measurement noise (Sinusoidal Noise of 1 RPM and Frequency $400 \mathrm{~Hz}$ ) and without noise are simulated for comparison. Descriptions are given in Table 3. Table 4 gives estimation errors for indicated torque observers described in Table 3. The mean estimation error is defined as $\int\left|\tilde{T}_{i}\right| d \theta / \int d \theta$. The SIO gives the best indicated torque estimation when there is no speed measurement noise. However, when high frequency measurement noise is present, the $\mathrm{CPO}$ gives the best estimation result. The SIO has estimation егто magnitudes about the same as the PIIO when high frequency noise is present. The advantage of using varying inertia over constant inertia for PIIO seems minimal.
Therefore, the use of varying inertia in the PI controller is not recommended. Estimation errors for various indicated torque observers are plotted in Figures 3 to 5 .

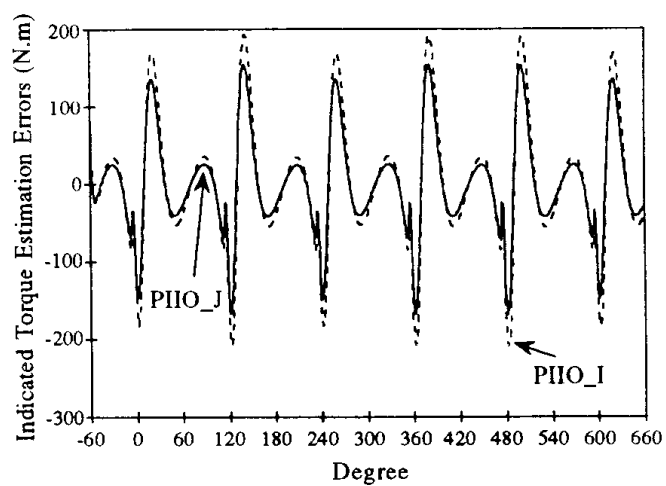

Figure 3: Indicated Torque Estimation Errors for PI Input Observer with Varying Inertia and with Constant Inertia (No Speed Measurement Noise).

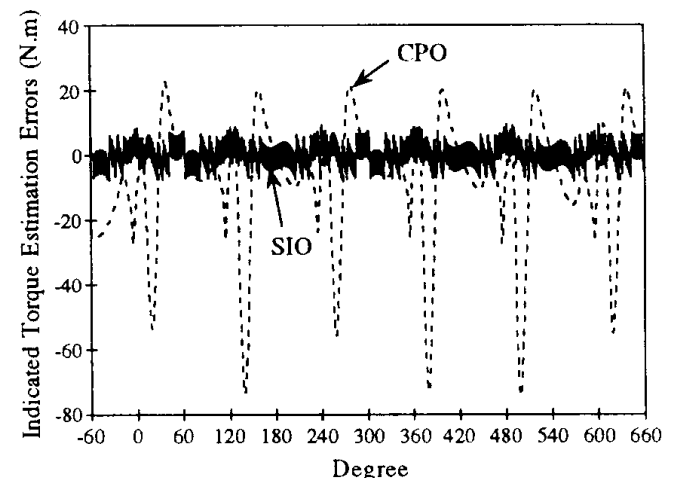

Figure 4: Indicated Torque Estimation Errors for Sliding Input Observer and Cylinder Pressure Observer (No Speed Measurement Noise).

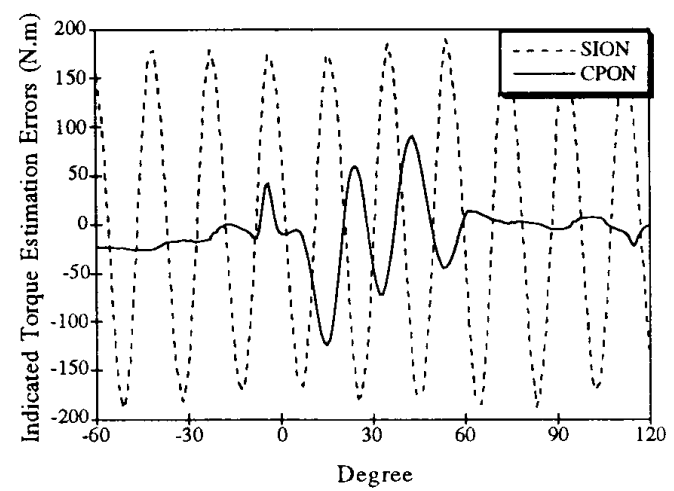

Figure 5: Indicated Torque Estimation Errors for Sliding Input Observer and Cylinder Pressure Observer (with Speed Measurement Noise).

A detailed cylinder-by-cylinder model is used to simulate a six-cylinder diesel engine. A misfire in the first cylinder and a half burn in the fifth cylinder are introduced momentarily 
during engine simulation. The cylinder pressure and varying speed are recorded. The cylinder pressure observer (CPO), PI feedforward input observer (PIIO), and sliding input observer (SIO) are employed to estimate either cylinder pressure or indicated torque.

Simulation results are shown in Figures 6 to 9. In Figure 6, the observed centroid is calculated from a cylinder pressure observer. The centroid of cylinder pressure for cylinder one is very close to zero or TDC. This strongly indicates a possible misfire. The cylinders two, three, four, and six have about the same locations of centroids. A normal combustion is predicted. However, cylinder five is questionable and indicated a partial burn. Notice that the observer algorithms do not have correct fuel burning information for the calculation and an identical fuel mass is intentionally introduced to all the cylinders in the simulation. All the observers have indicated the abnormal combustion in cylinders one and five. The mean indicated torques of all observers tested are average from TDC to 60 degree after TDC where combustion of one cylinder dominates. The results are given in Figure 7. The varying indicated torque estimated by a sliding input indicated torque observer is plotted in Figure 8. The peak indicated torques for every individual cylinder are also given in the figure. Abnormal combustion can also be predicted by using estimated peak indicated torques. The result of using the cylinder pressure difference approach is given in Figure 9. The observed cylinder pressures at 60 degree before and after TDC are compared. A pressure difference of close to zero for the first cylinder makes the misfire detection apparent. The partial burn is shown by a low pressure difference in the observed fifth cylinder.

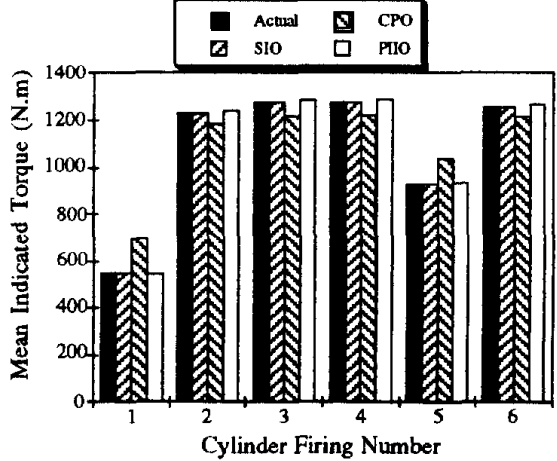

Figure 7: Simulated Actual and Observed Mean Indicated Torques from Various Indicated Torque Observers for a SixCylinder Engine.

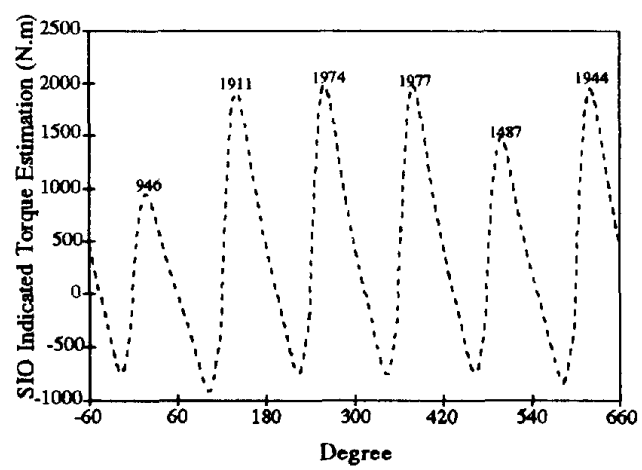

Figure 8: Indicated Torque Estimated by Sliding Input Observer for a Six-Cylinder Engine.

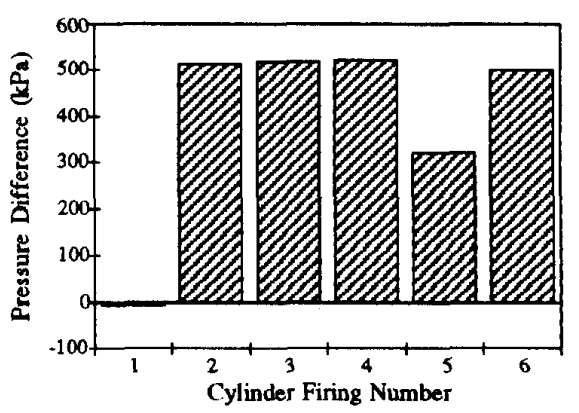

Figure 9: Observed Cylinder Pressure Difference $\Delta P_{\text {deg }}$ (Estimated by Cylinder Pressure Observer) for a Six-Cylinder Engine. 


\section{Summary}

This paper has described how to design input indicated torque observers that have good estimation accuracy. The sliding input observer has good performance, but is sensitive to noise. The varying speed is preferably filtered before use in these observers. Cylinder pressure and indicated torque estimates which come from these model-based observers are analyzed and related to combustion faults. The cylinder pressure observer estimates cylinder pressure for every individual cylinder, and provides cylinder pressure to centroid calculation. The centroid location can be easily used for misfire detection. Derivations of cylinder pressure difference approaches are discussed. Simulation results have shown that the average indicated torque of individual cylinder, peak indicated torque of individual cylinder, cylinder pressure differences, and cylinder pressure centroids can all be used to detect cylinder firing problem.

\section{Nomenclature}

$\wedge$ estimated value

$B D C$ bottom dead center

$J(\theta) \quad$ varying inertia of crankshaft

$\dot{m}_{\text {fburn }}$ fuel mass burning rate, $\mathrm{kg} / \mathrm{sec}$

$P_{c y l i} \quad$ ith cylinder pressure

$\dot{Q}_{h t} \quad$ heat transfer rate, $k W$

$Q_{L H V} \quad$ fuel lower heating value, $\mathrm{kJ} / \mathrm{kg}$

sgn(.) signum function

$T_{f} \quad$ friction torque, $N . m$

$T_{i} \quad$ indicated torque, $N . m$

$T_{\text {load }} \quad$ load torque, $N . m$

$V \quad$ cylinder volume, $\mathrm{m}^{3}$

$\alpha, k \quad$ observer gains

$\lambda, \eta \quad$ positive constant

$\theta \quad$ crank angle

$\gamma \quad$ specific heat ratio

$\omega \quad$ engine speed in $\mathrm{rad} / \mathrm{sec}$

$\omega_{m} \quad$ measured engine speed

\section{References}

[1] B. J. Challen, "Some Diesel Engine Sensors," SAE Paper 871628, 1987.

[2] P. M. Frank, "Fault Diagnosis in Dynamic Systems Using Analytical and Knowledge-Based Redundancy-A Survey and Some New Results," Automatica, Vol. 26, No. 3, pp. 459-474, 1990.

[3] P. M. Frank, "Fault Diagnosis in Dynamic Systems via State Estimation-A Survey." System Fault Diagnostics, Reliability and Related Knowledge-Based Approaches, Vol. 1, pp. 35-98, D. Reidel Publishing Company, 1987.

[4] J. C. Gilkey and J. D. Powell, "Fuel-Air Ratio Determination from Cylinder Pressure Time Histories," ASME Journal of Dynamic Systems, Measurement, and Control, Vol. 107, Dec., 1985.

[5] M. Kao, "Model-Based Turbocharged Diesel Engine Control and Diagnostics Using Nonlinear Sliding Control and Observers," Department of Mechanical Engineering, University of Wisconsin-Madison, Ph.D. Thesis, 1994.

[6] M. Kao and J. J. Moskwa, "Nonlinear turbocharged diesel engine control and state observation," ASME Advanced Automotive Technologies 1993, DSC-Vol. 52, Symposium on
Advanced Automotive Technologies: Advanced Engine Control Systems, ASME Winter Annual Meeting, New Orleans, Louisiana., November, 1993.

[7] M. Kao and J. J. Moskwa, "Turbocharged diesel engine modeling for nonlinear engine control and state estimation," ASME Advanced Automotive Technologies 1993, DSC-Vol. 52, Symposium on Advanced Automotive Technologies: Advanced Engine Control Systems, ASME Winter Annual Meeting, New Orleans, Louisiana., November, 1993.

[8] M. Kao and J. J. Moskwa, "Nonlinear cylinder and intake manifold pressure observers for engine control and diagnostics," SAE Paper 940375, SAE Special Publication SP1029 Electronic Engine Controls, Presented at $S A E$ International Congress \& Exposition, Detroit, Michigan, February, 1994.

[9] C. Ludwig, S. Leonhardt, M. Ayoubi, and R. Isermann, "Measurement and Monitoring of Pressure Curves in Diesel Engines," Proc. American Control Conference, San Francisco. California, June 1993.

[10] L. O'Connor, "Diagnosing Diesel Engines," Mechanical Engineering, pp. 44-50, March, 1992.

[11] R. J. Patton, P. M. Frank, and R. N. Clark, Fault Diagnosis in Dynamic Systems-Theory and Applications, Prentice-Hall International Ltd., 1989.

[12] B. K. Powell, G. P. Lawson, and G. Hogh, "Cylinder Pressure Feedback Control Analysis Model," Proc. American Control Conference, San Francisco, California, June 1993.

[13] J. D. Powell, "Engine Control Using Cylinder Pressure: Past, Present, and Future," ASME Journal of Dynamic Systems, Measurement, and Control, Vol. 115, No. 2B, 50th Anniversary Issue, pp. 343-350, June., 1993.

[14] K. Srinivasan, G. Rizzoni, M. Trigui, and G. Luh, "Online Estimation of Net Engine Torque from Crankshaft Angular Velocity Measurement Using Repetitive Estimators," Proc. American Control Conference, Chicago, IL, 1992.

[15] Y. Zhang and G. Rizzoni, "Design of Input Estimator for On-line Indicated Torque Estimation in IC engine Using Feedforward," ASME Winter Annual Meeting, New Orleans, La., Nov., 1993. 